\title{
Rhombencephalosynapsis with and without associated malformations
}

\author{
Kurt A. Jellinger
}

Received: 2 December 2008 / Accepted: 18 December 2008 / Published online: 10 January 2009

(C) Springer-Verlag 2009

Pasquier et al. [3], in a recent morphological analysis of 40 fetuses from 14 to 35 weeks of gestation found rhombencephalosynapsis (RES) in 11 cases and diencephalo-mesencephalosynapsis in 24 cases, usually associated with other brain anomalies or malformations (agenesis or hypoplasia of corpus callosum, lobar holoprosencephaly, encephalocele, stenosis or forking of Sylvian aqueduct, ventricular dilatation; and extraneuronal anomalies, e.g., VACTERL $+\mathrm{H}$ syndrome, etc), while in only two cases of RES (\#9, \#11) no additional abnormalities were observed. Thus, separation between pure neurological phenotypes and those associated with other syndrome-anomalies were possible.

These data can be confirmed in a small personal series of four cases of RES (two published previously [1]).

In a boy aged 7 years presenting with mental retardation and tetraparesis, in addition to typical RES no other CNS malformations were observed, while general autopsy revealed cardiac dilatation and pneumonia. In contrast, a girl aged 6 weeks, with deformation of the occipital cranium and keratomalacia but without neuro-psychiatric signs and symptoms, in addition to typical RES, showed a hypoplasia of the corpus callosum and pons, internal hydrocephalus and absence of the spinal trigeminal tracts. A boy aged 2 years with mental retardation, epileptic seizures and flaccid paraparesis of the legs, but without extraneuronal malformations, revealed typical RES, diffuse atrophy of the cerebral hemispheres, and fusion of both thalami. The oldest patient, a woman aged 26 years, with severe mental retardation, blindness, incontinence, severe behavioral disturbances, dying from pulmonary embolism, in the absence of extraneuronal malformations, showed typical RES, associated with severe internal hydrocephalus due to stenosis of the Sylvian aqueduct and consecutive atrophy of the optic nerves. Since in the first case of RES, reported by Obersteiner [2], no clinical data, general autopsy findings, nor exact data of the skull and brain are available, it cannot be further evaluated. Although no genetic examinations had been performed in our cases, together with those reported by Pasquier et al. [3], they suggest that the frequent combination of both telencephalic and hindbrain malformations in patients with RES may be due to disordered formation of the roof plate and development of midline cerebellar primordia at the junction of the mesencephalon and the first rhombomere.

\section{References}

1. Jellinger KA (2002) Rhombencephalosynapsis. Acta Neuropathol 103:305-306

2. Obersteiner H (1914) Ein Kleinhirn ohne Wurm. Arb Neurol Inst (Wien) 21:124-136

3. Pasquier L, Marcorelles P, Loget P, Pelluard F, Carles D, Perez MJ, Bendavid C, de La Rochebrochard C, Ferry M, David V, Odent S, Laquerrière A (2009) Rhombencephalosynapsis and related anomalies: a neuropathological study of 40 fetal cases. Acta Neuropathol (in press)
K. A. Jellinger $(\square)$

Institute of Clinical Neurobiology,

Kenyongasse 18, 1070 Vienna, Austria

e-mail: kurt.jellinger@univie.ac.at 